\title{
Concurrent Chemoradiotherapy Versus Radiation Monotherapy for Patients with Locally Advanced Esophageal Squamous Cell Carcinoma in the Era of Intensity Modulated Radiotherapy: A Propensity Score- matched Analysis
}

\section{Chen Li}

Department of Radiation Oncology, National Cancer Center/National Clinical Research Center for Cancer/Cancer Hospital, Chinese Academy of Medical Sciences and Peking Union Medical College Lijun Tan

Department of Oncology, First Affiliated Hospital of Harbin Medical College, Harbin 150000

Xiao Liu

Department of Radiation Oncology, Henan Cancer Hospital, Zhengzhou, 450000

\section{Xin Wang}

Department of Radiation Oncology, National Cancer Center/National Clinical Research Center for Cancer/Cancer Hospital, Chinese Academy of Medical Sciences and Peking Union Medical College

\section{Zongmei Zhou}

Department of Radiation Oncology, National Cancer Center/National Clinical Research Center for Cancer/Cancer Hospital, Chinese Academy of Medical Sciences and Peking Union Medical College

\section{Dongfu Chen}

Department of Radiation Oncology, National Cancer Center/National Clinical Research Center for Cancer/Cancer Hospital, Chinese Academy of Medical Sciences and Peking Union Medical College

\section{Qinfu Feng}

Department of Radiation Oncology, National Cancer Center/National Clinical Research Center for Cancer/Cancer Hospital, Chinese Academy of Medical Sciences and Peking Union Medical College Jun Liang

Department of Radiation Oncology, Cancer Hospital Chinese Academy of Medical Sciences, Shenzhen Center, Shenzhen, 518000

Jima Lv

Department of Radiation Oncology, National Cancer Center/National Clinical Research Center for Cancer/Cancer Hospital, Chinese Academy of Medical Sciences and Peking Union Medical College Xiaozhen Wang

Department of Radiation Oncology, National Cancer Center/National Clinical Research Center for Cancer/Cancer Hospital, Chinese Academy of Medical Sciences and Peking Union Medical College Nan Bi 
Department of Radiation Oncology, National Cancer Center/National Clinical Research Center for Cancer/Cancer Hospital, Chinese Academy of Medical Sciences and Peking Union Medical College

\section{Lei Deng}

Department of Radiation Oncology, National Cancer Center/National Clinical Research Center for Cancer/Cancer Hospital, Chinese Academy of Medical Sciences and Peking Union Medical College

\section{Wenqing Wang}

Department of Radiation Oncology, National Cancer Center/National Clinical Research Center for Cancer/Cancer Hospital, Chinese Academy of Medical Sciences and Peking Union Medical College

\section{Tao Zhang}

Department of Radiation Oncology, National Cancer Center/National Clinical Research Center for Cancer/Cancer Hospital, Chinese Academy of Medical Sciences and Peking Union Medical College

\section{Wenjie Ni}

Department of Radiation Oncology, National Cancer Center/National Clinical Research Center for Cancer/Cancer Hospital, Chinese Academy of Medical Sciences and Peking Union Medical College

\section{Xiao Chang}

Department of Radiation Oncology, National Cancer Center/National Clinical Research Center for Cancer/Cancer Hospital, Chinese Academy of Medical Sciences and Peking Union Medical College

\section{Weiming Han}

Department of Radiation Oncology, National Cancer Center/National Clinical Research Center for Cancer/Cancer Hospital, Chinese Academy of Medical Sciences and Peking Union Medical College

\section{Linrui Gao}

Department of Radiation Oncology, National Cancer Center/National Clinical Research Center for Cancer/Cancer Hospital, Chinese Academy of Medical Sciences and Peking Union Medical College

\section{Shijia Wang}

Department of Radiation Oncology, National Cancer Center/National Clinical Research Center for Cancer/Cancer Hospital, Chinese Academy of Medical Sciences and Peking Union Medical College

\section{Zefen Xiao ( $\nabla$ xiaozefen@sina.com )}

Department of Radiation Oncology, National Cancer Center/National Clinical Research Center for Cancer/Cancer Hospital, Chinese Academy of Medical Sciences and Peking Union Medical College

\section{Research Article}

Keywords: Esophageal cancer, Definitive radiotherapy, Concurrent chemoradiotherapy, Intensity-modulated radiotherapy, Propensity score-matched

Posted Date: December 17th, 2020

DOI: https://doi.org/10.21203/rs.3.rs-125831/v1

License: (c) (i) This work is licensed under a Creative Commons Attribution 4.0 International License. Read Full License 


\section{Abstract}

Background: To investigate the survival benefit of concurrent chemoradiotherapy for patients with locally advanced esophageal squamous cell carcinoma during the years of intensity-modulated radiotherapy.

Methods: Medical records of 1273 patients with esophageal squamous cell carcinoma who received intensitymodulated radiotherapy from January 2005 to December 2017 in the CAMS were retrospectively reviewed. 683 patients received concurrent chemoradiotherapy, 590 patients received radiotherapy alone. Propensity score matching (PSM) method was used to eliminate baseline differences between the two groups. Survival and toxicity profile were evaluated afterwards.

Results: After a median follow-up time of 50.4 months (3.2-157.4 months), both overall survival and progression-free survival of the concurrent chemoradiotherapy group were better than those of the radiotherapy group, either before or after PSM. After PSM, the 1-, 3-, 5-year OS of radiotherapy and concurrent chemoradiotherapy groups were $63.3 \%$ vs $72.2 \%, 31.6 \%$ vs $42.2 \%$ and $28.5 \%$ vs $38.1 \%$, respectively $(p=0.003)$. The 1-year, 3-year and 5-year PFS rates of radiotherapy and concurrent chemoradiotherapy group were $44.3 \%$ vs $48.6 \%, 23.4 \%$ vs $31.2 \%$ and $15.8 \%$ vs $25.2 \%$, respectively ( $p=0.002$ ). The rates of $\geq$ grade 3 leukopenia and radiation esophagitis in the concurrent chemoradiotherapy were higher than those in the radiotherapy alone group $(p<0.05)$. There was no significant difference in the probability of radiation pneumonia between the two groups ( $p=0.359$ ). Multivariate logistic regression analysis showed $\geq 70$ years old, female, KPS $\leq 70$, stage I-II, and patients diagnosed at earlier years (2005-2010) had lower probability of receiving concurrent chemoradiation $(p<0.05)$. Multivariate Cox analysis indicated that female, stage $\mathrm{H}-\mathrm{II}, \mathrm{EQD} 2 \geq 60 \mathrm{~Gy}$ and concurrent chemotherapy were favorable prognostic factors for both OS and PFS.

Conclusions: Concurrent chemotherapy can bring survival benefits to patients with locally advanced esophageal squamous cell carcinoma receiving intensity-modulated radiotherapy. For patients who cannot tolerate concurrent chemotherapy, radiation monotherapy is an effective alternative with promising results.

\section{Background}

Esophageal cancer (EC) is a common malignancy with poor prognosis, and China is the country with the largest number of new cases and deaths every year. According to a national epidemiological research conducted in 2015[1], the numbers of new diagnosed cases and deaths of esophageal cancer in China have reached 478,000 and 375,000 , ranking third and fourth respectively among all malignant tumors. Unlike most Western countries, the pathological type of esophageal cancer in China is still dominated by squamous cell carcinoma (SCC), and patients with esophageal squamous cell carcinoma account for more than $90 \%$ of all EC patients[2, 3].

Due to insufficient promotion of annual endoscopy screening, a certain portion of patients in China present with advanced disease at diagnosis and are not suitable for esophagectomy. For these patients, definitive concurrent chemoradiotherapy (CCRT) has become the standard of care as recommended by most guidelines since the RTOG 85 - 01 study[4-6]. However, in clinical practice, a considerable number of patients could not tolerate concurrent chemoradiation for various reasons (i.e., advanced age, poor general condition, poor 
nutritional status, obvious weight loss, patient refusion, etc.). For this group of patients, the most commonly used treatment is radiation monotherapy.

In the RTOG85-01 study, the 5-year survival rate of patients in the radiotherapy-only group was just 0 , which indicates that radiation monotherapy is very ineffective in treating locally advanced esophageal cancer. According to the clinical experience and some contemporary large retrospective analysis results[7-9], however, the survival outcome of patients receiving radiotherapy alone is much better than that of RTOG $85-01$. Besides, as a research started in the 1980s, RTOG 85 - 01 employed the conventional two-dimensional radiotherapy (2DRT), whereas radiation techniques have been evolving rapidly in last few decades. The emergence of three-dimensional conformal radiotherapy (3DCRT) and intensity-modulated radiotherapy (IMRT) has greatly improved the conformality of dose distribution, making it possible to increase the dose of the planning target volume without increasing the irradiation dose to adjacent normal tissues[10]. Many studies have shown that advanced radiation techniques have reduced the incidence of side effects and improved the local control of esophageal cancer patients[11]. Unfortunately, there are very limited reports on the survival and prognosis of patients receiving radiation monotherapy using the aforementioned advanced radiation technique (i.e., IMRT), let alone studies comparing the survival results of patients receiving radiotherapy alone with that of patients receiving CCRT.

Therefore, we conducted this retrospective study to collect data of patients receiving IMRT alone and compare with the survival of patients receiving CCRT in the same period, in the hope of providing a baseline reference of treatment standard and prognosis for patients with advanced esophageal cancer.

\section{Method}

\section{Patient eligibility}

A total of 2,257 patients who did not receive surgery and received IMRT at the Cancer Hospital of the Chinese Academy of Medical Sciences from 2005 to 2017 were included. Patients were excluded if they had: (1) missing clinical data $(n=22)$; (2) missing follow-up data $(n=23)$ or $<3$ months follow-up time $(n=67)$; (3) distant visceral metastasis at initial diagnosis $(n=109)$; $(4)$ prior active malignancies (other than curable nonmelanoma skin cancer or in situ cervical cancer) within 5 years $(n=109)$; (5) received sequential chemotherapy (either before or after radiation monotherapy) $(n=124)$; (6) received concurrent molecular target drugs $(n=$ 131); (7) received palliative treatment (radiation dose < 40 Gy or palliative radiation field) $(n=54)$; (8) histological types other than squamous cell carcinoma (e.g., adenocarcinoma, small cell carcinoma, and so on; $n=55$ ). After exclusions, 1273 patients stayed in the final analysis cohort (CCRT $=683$, RT alone $=590)$ (Fig. 1). Staging was determined based on the 6th edition (2002) of the American Joint Committee on Cancer TNM staging.

\section{Treatment}

All patients received computed tomography (CT) simulation and IMRT. The gross tumor volume (GTV-T) was defined as the primary tumor. The GTV-T would be determined using all available resources (physical examination, upper gastrointestinal contrast, endoscopy, EUS, contrast CT-thorax/abdomen, contrast MRIthorax/abdomen, PET-CT, etc.). The gross lymph nodes volume (GTV-N) were defined as any lymph node 
diagnosed as or highly-suspected as metastatic. The clinical target volume (CTV) comprised of a $0.5-0.8 \mathrm{~cm}$ lateral margin and a $3.0 \mathrm{~cm}$ longitudinal margin around GTV, a $0.5 \mathrm{~cm}$ uniform margin around GTVnd, and relevant lymphatic drainage areas. Planning target volume (PTV) was derived from CTV plus a uniform $0.5 \mathrm{~cm}$ margin. The boost volume (PGTV) was created by expanding GTV-T by $1.0 \mathrm{~cm}$ craniocaudally and $0.5 \mathrm{~cm}$ radially, and the GTV-N by a uniform $0.5 \mathrm{~cm}$ margin.

The median dose of CCRT and RT alone group was 59.92 Gy (range: 40.0-70.0 Gy) and 60.0 Gy (range: 40.0$70.0 \mathrm{~Gy}$ ), respectively. The prescription to PTV varies from $1.8 \mathrm{~Gy} /$ fraction to $2.2 \mathrm{~Gy} /$ fraction whereas the prescription to PGTV varies from $2.0 \mathrm{~Gy} /$ fraction to $2.4 \mathrm{~Gy} /$ fraction ( 1 fraction per day, 5 fractions per week). As the fractionation schemes have slight variations between patients, we converted the radiation dose of each patient into equivalent dose in $2.0 \mathrm{~Gy} /$ fration (EQD2) in the final analysis.

All patients received CT simulation, and their image data were registered in the treatment planning system (Pinnacle; Philips Medical Systems, Hanover, MA, USA).

It is required that at least $95 \%$ of the PTV received the prescribed dose. Dose volume histograms (DVH) was adopted to assess the quality of the treatment plan and the exposure of organs at risk (OAR). The volume of lung tissue receiving 20 Gy or more should not exceed $28 \%$ of the total lung volume (V20 lung $<28 \%$ ). The mean dose of lung tissue should be lower than $16 \mathrm{~Gy}$ (Dmean lung < 16Gy). Other dose constraints to OARs include: V40 heart < 30\%, V30 heart < 40\%, V40 stomach < 40\%, Dmax stomach < 55-60Gy, V40 small intestine < $40 \%$, Dmax small intestine < 55Gy, V30 liver < 30\%, V20 kidney < 30\% and Dmax spinal cord PRV < 45 Gy. Imageguided radiotherapy (IGRT) was applied to all patients either by electronic portal imaging device (EPID) or cone beam computed tomography (CBCT).

For patients receiving CCRT, the most commonly used concurrent chemotherapeutic regimen was the combination of taxane and platinum-based compounds ( $n=534,78.2 \%)$, followed by the combination of fluorouracil and platinum- based compounds $(n=62,9.1 \%)$, and some patients also received single-agent fluorouracil $(n=70,10.2 \%)$ or platinum $(n=12,1.8 \%)$ for concurrent chemotherapy.

The most common reasons for not receiving concurrent chemotherapy include: 1) obvious weight loss before treatment, weak general condition, poor performance status; 2) elderly patients; 3) serious cardiopulmonary complications or insufficient hepatic or renal functions; 4) patients' or relatives' refusal.

\section{Outcome measures}

Acute and late toxicities were scored according to the Common Toxicity Criteria for Adverse Events, version 4.0[12]. In brief, patients were assessed every 3 months for the first 2 years after RT, every 6 months for the next 3 years, and then once annually. Assessments included barium esophagram; CT of neck, chest and upper abdomen with contrast; ultrasonography of the neck and upper abdomen; and conventional blood and biochemistry studies. Positron emission tomography-CT (PET-CT) and fine-needle aspiration cytology were performed if needed. Bone scan was performed in case of bone pain or abnormally elevated serum alkaline phosphatase (ALP), and cranial MRI was performed if clinically indicated.

Overall Survival (OS) was measured as the interval between the beginning of RT to the date of death from any cause or final follow-up. Progression-free survival (PFS) was defined as the interval between the end of RT and 
the date of first recurrence or death from any cause. Patients who had not experienced progression or death by the last follow-up were administratively censored.

\section{Statistical Analysis}

Descriptive statistics, including frequencies and percentages for categorical variables, and mean and standard deviation for quantitative variables, were computed to summarize patient characteristics for the entire cohort and for each treatment group. Between-group comparisons to evaluate imbalances in covariates were conducted using t-tests and Chi-square test for quantitative and categorical variables respectively. The incidence of toxicities was compared by Chi-square test and Fisher's exact test.

OS time and PFS time were estimated for RT alone and CCRT group using Kaplan- Meier (KM) plots both before and after PSM. Log-rank test was carried out to compare event time distributions between two groups. A Cox regression model was used to perform multivariate analyses of the effect of covariates on OS and PFS after PSM. The results of the Cox models were expressed as hazard ratios (HRs) along with the $95 \% \mathrm{Cl}$ estimates. Multivariable logistic regression models were used to identify factors associated with undergoing concurrent chemotherapy. The results of these logistic regression analyses were expressed as adjusted odds ratios (ORs) along with the corresponding $95 \%$ confidence intervals (95\% Cls).

To adjust unbalanced covariates, PSM[13] method was used. The propensity score for each patient was estimated with a logit model that included the following variables: age, sex, KPS, TNM stage, tumor location, radiation dose and the year of diagnosis. Exact matching method was performed on TNM stage and sex whereas nearest matching method was adopted for the remaining variables. Setting caliper $=0.10$, matching ratio $=1: 1$, two comparable groups of patients were created with 294 patients in each group. The significance level was set as p-value less than 0.05. All computations were conducted in R 2.13.0.

\section{Results}

\section{Patient and treatment characteristics}

A total of 1273 patients were involved in this study, including 590 patients in the CCRT group and 683 patients in the RT alone group. The patient, tumor, and treatment characteristics were summarized in Table 1. 
Table 1

Patient, tumor and treatment characteristics before and after PSM

\begin{tabular}{|c|c|c|c|c|c|c|c|c|c|c|c|c|}
\hline \multirow[t]{3}{*}{ Variables } & & & \multicolumn{5}{|c|}{ Before Matching } & \multicolumn{5}{|c|}{ After Matching } \\
\hline & \multicolumn{2}{|c|}{ Overall } & \multicolumn{2}{|c|}{ RT alone } & \multicolumn{3}{|c|}{ CCRT } & \multicolumn{2}{|c|}{ RT alone } & \multicolumn{3}{|c|}{ CCRT } \\
\hline & $\begin{array}{l}n= \\
1273\end{array}$ & $\%$ & $\begin{array}{l}\mathrm{n}= \\
590\end{array}$ & $\%$ & $\begin{array}{l}n= \\
683\end{array}$ & $\%$ & $p$ & $\begin{array}{l}n= \\
294\end{array}$ & $\%$ & $\begin{array}{l}n= \\
294\end{array}$ & $\%$ & $p$ \\
\hline Age & \multicolumn{2}{|c|}{$63(29-89)$} & \multicolumn{2}{|c|}{$70(40-89)$} & \multicolumn{2}{|c|}{$59(33-81)$} & 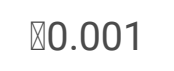 & \multicolumn{2}{|c|}{$61(40-69)$} & \multicolumn{2}{|c|}{$61(33-69)$} & 1.000 \\
\hline$<70 y$ & 888 & 69.8 & 259 & 43.9 & 629 & 92.1 & & 243 & 82.7 & 243 & 82.7 & \\
\hline$\geq 70 y$ & 385 & 30.2 & 331 & 56.1 & 54 & 7.9 & & 51 & 17.3 & 51 & 17.3 & \\
\hline Sex & & & & & & & $\otimes 0.001$ & & & & & 1.000 \\
\hline male & 1041 & 81.8 & 451 & 76.4 & 590 & 86.4 & & 241 & 82.0 & 241 & 82.0 & \\
\hline female & 232 & 18.2 & 139 & 23.6 & 93 & 13.6 & & 53 & 18.0 & 53 & 18.0 & \\
\hline KPS & & & & & & & $\otimes 0.001$ & & & & & 0.666 \\
\hline$\leq 70$ & 122 & 9.6 & 95 & 16.1 & 27 & 4.0 & & 28 & 9.5 & 25 & 8.5 & \\
\hline$>70$ & 1151 & 90.4 & 475 & 83.9 & 656 & 96.0 & & 266 & 90.5 & 269 & 91.5 & \\
\hline $\begin{array}{l}\text { Tumor } \\
\text { location }\end{array}$ & & & & & & & 0.271 & & & & & 0.862 \\
\hline cervical & 52 & 3.7 & 21 & 3.7 & 21 & 3.5 & & 10 & 3.8 & 12 & 4.6 & \\
\hline upper & 415 & 29.5 & 158 & 27.6 & 193 & 32.4 & & 83 & 31.7 & 84 & 32.1 & \\
\hline middle & 689 & 49.0 & 296 & 51.7 & 277 & 46.6 & & 131 & 50.0 & 123 & 46.9 & \\
\hline lower & 250 & 17.8 & 97 & 17.0 & 104 & 17.5 & & 38 & 14.5 & 43 & 16.4 & \\
\hline T stage & & & & & & & 0.202 & & & & & 0.841 \\
\hline T1 & 42 & 3.3 & 23 & 3.9 & 19 & 2.8 & & 10 & 3.4 & 7 & 2.4 & \\
\hline T2 & 112 & 8.8 & 57 & 9.7 & 55 & 8.1 & & 29 & 9.9 & 31 & 10.5 & \\
\hline Т3 & 575 & 45.2 & 274 & 46.4 & 301 & 44.1 & & 127 & 43.2 & 133 & 45.2 & \\
\hline T4 & 542 & 42.6 & 236 & 40.0 & 306 & 44.8 & & 128 & 43.5 & 123 & 41.8 & \\
\hline TX & 2 & 0.2 & 0 & 0.0 & 2 & 0.3 & & 0 & 0.0 & 0 & 0.0 & \\
\hline N stage & & & & & & & $\otimes 0.001$ & & & & & 0.122 \\
\hline NO & 181 & 14.2 & 124 & 21.0 & 57 & 8.3 & & 43 & 14.6 & 28 & 9.5 & \\
\hline N1 & 1088 & 85.5 & 464 & 78.6 & 624 & 91.4 & & 249 & 84.7 & 265 & 90.1 & \\
\hline NX & 4 & 0.3 & 2 & 0.3 & 2 & 0.3 & & 2 & 0.7 & 1 & 0.3 & \\
\hline
\end{tabular}




\begin{tabular}{|c|c|c|c|c|c|c|c|c|c|c|c|c|}
\hline \multirow{2}{*}{\begin{tabular}{|l|} 
Variables \\
M stage
\end{tabular}} & & & \multicolumn{5}{|c|}{ Before Matching } & \multicolumn{5}{|c|}{ After Matching } \\
\hline & & & & & & & $\varangle 0.001$ & & & & & 0.993 \\
\hline MO & 957 & 75.2 & 490 & 83.1 & 467 & 68.4 & & 230 & 78.2 & 229 & 77.9 & \\
\hline M1a & 120 & 9.4 & 35 & 5.9 & 85 & 12.4 & & 20 & 6.8 & 20 & 6.8 & \\
\hline M1b & 196 & 15.4 & 65 & 11.0 & 131 & 19.2 & & 44 & 15.0 & 45 & 15.3 & \\
\hline $\begin{array}{l}\text { TNM } \\
\text { stage } \\
\text { (6th ) }\end{array}$ & & & & & & & $₫ 0.001$ & & & & & 1.000 \\
\hline$|-I|$ & 184 & 14.5 & 118 & 20.0 & 66 & 9.7 & & 41 & 13.9 & 41 & 13.9 & \\
\hline III & 772 & 60.6 & 371 & 62.9 & 401 & 58.7 & & 188 & 63.9 & 188 & 63.9 & \\
\hline IV & 317 & 24.9 & 101 & 17.1 & 216 & 31.6 & & 65 & 22.1 & 65 & 22.1 & \\
\hline $\begin{array}{l}\text { Year of } \\
\text { diagnosis }\end{array}$ & & & & & & & $\otimes 0.001$ & & & & & 0.778 \\
\hline $\begin{array}{l}2005- \\
2010\end{array}$ & 283 & 22.2 & 174 & 29.5 & 109 & 16.0 & & 75 & 25.5 & 78 & 26.5 & \\
\hline $\begin{array}{l}2011- \\
2017\end{array}$ & 990 & 77.8 & 416 & 60.5 & 574 & 84.0 & & 219 & 74.5 & 216 & 73.5 & \\
\hline EQD2 & & & & & & & 0.251 & & & & & 0.224 \\
\hline$<60 \mathrm{~Gy}$ & 330 & 25.9 & 144 & 24.4 & 186 & 27.2 & & 71 & 24.1 & 84 & 28.6 & \\
\hline$\geq 60$ Gy & 943 & 74.1 & 446 & 75.6 & 497 & 72.8 & & 223 & 75.9 & 210 & 71.4 & \\
\hline
\end{tabular}

Patients in CCRT group were more likely to be younger (59 years old [median age] for CCRT vs 70 years old [median age] for RT alone, $p<0.001)$, male ( $86.4 \%$ for CCRT vs $76.4 \%$ for RT alone, $p<0.001)$, to have better performance status ( $4.0 \%$ KPS $\leq 70$ for CCRT vs $16.1 \%$ for RT alone, $p<0.001)$, to have more $\mathrm{N}+$ stage $(91.4 \%$ vs $78.6 \%, p<0.001), M+$ stage ( $M+31.6 \%$ vs $16.9 \%, p<0.001)$ and advanced TNM stage (stage III-IV $90.3 \%$ vs $80.0 \%, p<0.001)$ and to be diagnosed at more recent times $(2011-201784.0 \%$ vs $60.5 \%, p<0.001)$.

The propensity score-matched cohort included 294 patients in the CCRT group and 294 patients in the RT alone group, and all the selected covariates were well-balanced between the two matched groups (see Table 1).

\section{Survival Results}

The median follow-up time of the whole cohort of surviving patients was 50.4 months (3.2 157.4 months), of which 49.8 months (3.2 149.8 months) in the CCRT group and 52.1 months (4.7 157.4 months) in the RT alone. Median OS time of the entire cohort was 20.9 months (95\% Cl: 19.3-22.5 months), and the median PFS time was 11.5 months ( $95 \% \mathrm{Cl} 10.3-12.8$ months). For patients receiving RT alone, the 1-year, 3-year, and 5year OS rates before PSM were $65.3 \%, 32.6 \%$, and $24.8 \%$, respectively. The 1-year, 3-year, and 5-year PFS rates were $46.4 \%, 22.9 \%$ and $15.9 \%$, respectively. 
The addition of concurrent chemotherapy has further improved both OS and PFS. Figure 2 presents the survival curves comparing RT alone with CCRT both before and after PSM. Compared with the RT alone group, the CCRT group had better OS and PFS rates whether before or after PSM (before-match OS: log-rank $p<0.001$ [Fig. 2A]; after-match OS: log-rank $p=0.003$ [Fig. 2B]; before-match PFS: log-rank $p=0.003$ [Fig. 2C]; after-match PFS: log-rank $p=0.02$ [Fig. 2D];).

Among the 294 matched pairs in the PSM sample, the adjusted median OS was longer in the CCRT group (24.2 months; 95\% Cl, 18.9-29.5 months) compared with the RT alone group (17.7 months; 95\% Cl, 14.820.5 months). The adjusted median PFS was also longer in the CCRT group (11.4 months; $95 \% \mathrm{Cl}$, 8.414.4 months) compared with the RT alone group (8.7 months; $95 \% \mathrm{Cl}$, 6.3-11.0 months). In the after-matched cohort, the 1-year, 3-year and 5-year OS rates of RT alone and CCRT group were $63.3 \%$ vs $72.2 \%, 31.6 \%$ vs $42.2 \%$ and $28.5 \%$ vs $38.1 \%$, respectively. As for PFS rates, the 1-year, 3-year and 5-year PFS rates of RT alone and CCRT group were $44.3 \%$ vs $48.6 \%, 23.4 \%$ vs $31.2 \%$ and $15.8 \%$ vs $25.2 \%$, respectively.

\section{Subgroup Analysis}

To further explore the potential beneficiaries of concurrent chemotherapy, we conducted several subgroup analyses of OS in the PSM sample. Results showed that male $(p=0.004)$, age $<70$ years $(p=0.001)$, stage III-IV $(p=0.004), \mathrm{EQD} 2 \geq 60$ Gy $(p=0.004)$, and patients diagnosed between 2011-2017 $(p=0.006)$ could benefit from concurrent chemoradiotherapy. The subgroup analysis of age and radiation dose is shown in Fig. 3. Among the 588 paired patients, the vast majority were non-elderly patients $(n=486)$, and most patients received high-dose radiotherapy $(n=433)$. Among 102 elderly patients (aged over 70 years) in the matched sample, CCRT did not show survival benefit over RT alone $(p=0.8)$. In non-elderly patients, the OS rate of the CCRT group was better than that of the RT alone group $(p<0.001)$. Among 155 patients receiving low-dose radiotherapy (EQD2 <60 Gy) in the matched sample, CCRT did not show survival benefit over RT alone $(p=$ 0.12). In high-dose group, the OS rate of the CCRT group was better than that of the RT alone group $(p=0.004)$.

\section{Multivariate Analyses in after-PSM Cohort}

The multivariate Cox regression analysis for OS and PFS after PSM is summarized in Table 2. Factors that related to promising survival included female (OS: $\mathrm{HR}=0.61,95 \% \mathrm{Cl}: 0.46-0.81, p=0.001 ; \mathrm{PFS}: \mathrm{HR}=0.66,95 \%$ Cl: 0.51-0.86, $p=0.002)$, concurrent chemotherapy (OS: $\mathrm{HR}=0.68,95 \% \mathrm{Cl}: 0.56-0.83, p<0.001 ; \mathrm{PFS}: \mathrm{HR}=$ 0.78, 95\% Cl: $0.64-0.93, p=0.007)$ and EQD2 $\geq 60$ Gy (OS: HR = 0.64, 95\% Cl: 0.51-0.80, $p<0.001 ; \mathrm{PFS}: \mathrm{HR}=$ $0.68,95 \% \mathrm{Cl}: 0.55-0.83, p<0.001)$. Factors that related to inferior survival was advanced TNM stage. Survival did not differ by age (<70y vs $\geq 70 y)$, KPS, tumor location or year of diagnosis. 
Table 2

Results of multivariate Cox analysis for PFS and OS in the after-PSM cohort

\begin{tabular}{|c|c|c|c|c|}
\hline Variables & PFS & & os & \\
\hline & $\mathrm{HR}(95 \% \mathrm{Cl})$ & $p$ & $\mathrm{HR}(95 \% \mathrm{Cl})$ & $p$ \\
\hline Age & & 0.208 & & 0.653 \\
\hline$<70 y$ & - & - & - & - \\
\hline$\geq 70 y$ & $0.85(0.66-1.10)$ & - & $0.94(0.72-1.23)$ & \\
\hline Sex & & 0.002 & & 0.001 \\
\hline Male & - & - & - & - \\
\hline Female & $0.66(0.51-0.86)$ & - & $0.61(0.46-0.81)$ & - \\
\hline KPS & & 0.402 & & 0.350 \\
\hline$\leq 70$ & - & - & - & - \\
\hline$>70$ & $0.88(0.64-1.19)$ & - & $0.86(0.62-1.19)$ & - \\
\hline Tumor location & & 0.638 & & 0.538 \\
\hline Cervical & - & - & - & - \\
\hline Upper & $0.94(0.54-1.64)$ & 0.834 & $0.96(0.54-1.71)$ & 0.902 \\
\hline Middle & $0.85(0.49-1.48)$ & 0.561 & $0.87(0.49-1.54)$ & 0.626 \\
\hline Lower & $0.98(0.55-1.75)$ & 0.945 & $1.05(0.58-1.92)$ & 0.868 \\
\hline TNM Stage (6th ) & & $<0.001$ & & $<0.001$ \\
\hline I-II & - & - & - & - \\
\hline III & $1.92(1.40-2.63)$ & $<0.001$ & $1.96(1.38-2.79)$ & $<0.001$ \\
\hline IV & $2.77(1.95-3.94)$ & $<0.001$ & $2.78(1.90-4.37)$ & $<0.001$ \\
\hline Y of diagnosis & & - & & 0.417 \\
\hline 2005-2010 & - & - & - & - \\
\hline 2011-2016 & $1.10(0.87-1.39)$ & 0.040 & $1.10(0.87-1.39)$ & - \\
\hline EQD2 & & $<0.001$ & & $<0.001$ \\
\hline$<60 G y$ & - & - & - & - \\
\hline$\geq 60 \mathrm{~Gy}$ & $0.68(0.55-0.83)$ & - & $0.64(0.51-0.80)$ & - \\
\hline Concurrent Chemotherapy & $0.78(0.64-0.93)$ & 0.007 & $0.68(0.56-0.83)$ & $<0.001$ \\
\hline
\end{tabular}

\section{Toxicities}


We further compared the profile of treatment-related toxicities between RT alone and CCRT group in the matched cohort. As shown in Table 3, the incidences of $\geq$ grade 2 anemia, thrombocytopenia, $\geq$ grade $2 / 3$ leukopenia and radiation esophagitis in the CCRT group were significantly higher than those in the RT alone group (all of which $p<0.05$ ). The most common Grade 3-4 toxicities were leucopenia $(13.7 \%)$ and radiation esophagitis (11.3\%) in the CCRT group, while the most common Grade 3-4 toxicity in the RT alone group was radiation esophagitis (5.0\%). There were six and three cases of treatment-related deaths in the CCRT and RT alone group respectively, all of which were attributed to Grade 5 radiation pneumonitis. No significant difference was seen in the incidences of radiation pneumonitis between the two groups $(p=0.36)$.

Table 3

Treatment-related toxicities of RT alone and CCRT groups in the after-PSM cohort

\begin{tabular}{|c|c|c|c|c|c|c|c|}
\hline \multirow[t]{2}{*}{ Toxicities } & \multicolumn{3}{|l|}{ RT alone } & \multicolumn{3}{|l|}{ CCRT } & \multirow[t]{2}{*}{$p$} \\
\hline & Grade1-2 & Grade3-4 & Grade5 & Grade1-2 & Grade3-4 & Grade 5 & \\
\hline Anemia & $15.4 \%$ & $0.3 \%$ & $0.0 \%$ & $21.9 \%$ & $1.4 \%$ & $0.0 \%$ & 0.040 \\
\hline$\geq$ Grade 2 & $2.7 \%$ & & & $6.5 \%$ & & & 0.031 \\
\hline$\geq$ Grade 3 & $0.3 \%$ & & & $1.4 \%$ & & & 0.216 \\
\hline Leukopenia & $44.7 \%$ & $1.0 \%$ & $0.0 \%$ & $67.5 \%$ & $13.7 \%$ & $0.0 \%$ & 0.000 \\
\hline$\geq$ Grade 2 & $13.7 \%$ & & & $53.8 \%$ & & & 0.000 \\
\hline$\geq$ Grade 3 & $1.0 \%$ & & & $13.7 \%$ & & & 0.000 \\
\hline Thrombocytopenia & $8.5 \%$ & $0.7 \%$ & $0.0 \%$ & $23.0 \%$ & $2.4 \%$ & $0.0 \%$ & 0.000 \\
\hline$\geq$ Grade 2 & $3.4 \%$ & & & $10.3 \%$ & & & 0.001 \\
\hline$\geq$ Grade 3 & $0.7 \%$ & & & $2.4 \%$ & & & 0.106 \\
\hline Esophagitis & $69.8 \%$ & $5.0 \%$ & $0.0 \%$ & $74.2 \%$ & $11.3 \%$ & $0.0 \%$ & 0.000 \\
\hline$\geq$ Grade 2 & $39.3 \%$ & & & $55.0 \%$ & & & 0.000 \\
\hline$\geq$ Grade 3 & $7.7 \%$ & & & $13.4 \%$ & & & 0.030 \\
\hline Skin Reaction & $50.2 \%$ & $2.2 \%$ & $0.0 \%$ & $63.5 \%$ & $2.4 \%$ & $0.0 \%$ & 0.007 \\
\hline$\geq$ Grade 2 & $14.7 \%$ & & & $20.6 \%$ & & & 0.071 \\
\hline$\geq$ Grade 3 & $2.2 \%$ & & & $2.5 \%$ & & & 0.830 \\
\hline Pneumonitis & $1.8 \%$ & $0.7 \%$ & $1.1 \%$ & $3.6 \%$ & $1.4 \%$ & $2.1 \%$ & 0.359 \\
\hline$\geq$ Grade 2 & $2.2 \%$ & & & $4.7 \%$ & & & 0.106 \\
\hline$\geq$ Grade 3 & $1.8 \%$ & & & $3.6 \%$ & & & 0.195 \\
\hline
\end{tabular}

Factors Associated with Receipt of Concurrent Chemotherapy 
Factors found to be significantly associated with undergoing concurrent chemotherapy were assessed using a multivariable logistic regression analysis that included all previously mentioned covariates as shown in Table 4. Results showed that factors affecting patients receiving CCRT include age $(p<0.001)$, sex $(p=0.02)$, KPS $(p<0.001)$, TNM stage $(p<0.001)$, and year of diagnosis $(p<0.001)$. Female patients, patients aged $\geq 70$ years, patients with a KPS $\leq 80$, patients with stage I or II disease, and those diagnosed at earlier times (2005$2010)$ were found to be less likely to receive concurrent chemotherapy. Tumor location $(p=0.502)$ and radiation dose $(p=0.183)$ did not show a significant correlation with the receipt of concurrent chemotherapy. 
Table 4

Multivariable Logistic Regression Analysis of Factors Associated with Receipt of Concurrent Chemotherapy

\begin{tabular}{|c|c|c|c|}
\hline Variables & CCRT rate & OR (95\% Cl) & $p$ \\
\hline Age & & & $<0.001$ \\
\hline$<70 y$ & $75.1 \%$ & - & - \\
\hline$\geq 70 y$ & $60.3 \%$ & $0.075(0.054-0.105)$ & - \\
\hline Sex & & & 0.022 \\
\hline Male & $69.7 \%$ & - & - \\
\hline Female & $58.0 \%$ & $0.660(0.462-0.941)$ & - \\
\hline KPS & & & $<0.001$ \\
\hline$\leq 70$ & $37.9 \%$ & - & - \\
\hline$>70$ & $69.9 \%$ & $3.537(2.110-5.931)$ & - \\
\hline Tumor location & & & 0.502 \\
\hline Cervical & $65.7 \%$ & - & - \\
\hline Upper & $67.9 \%$ & $1.044(0.488-2.233)$ & 0.912 \\
\hline Middle & $66.2 \%$ & $0.851(0.400-1.808)$ & 0.674 \\
\hline Lower & $73.6 \%$ & $1.074(0.479-2.408)$ & 0.862 \\
\hline TNM Stage (6th ) & & & $<0.001$ \\
\hline I-II & $60.2 \%$ & - & - \\
\hline III & $65.8 \%$ & $1.582(1.059-2.363)$ & 0.025 \\
\hline IV & $75.1 \%$ & $2.684(1.693-4.257)$ & $<0.001$ \\
\hline Y of diagnosis & & & $<0.001$ \\
\hline $2005-2010$ & $52.8 \%$ & - & - \\
\hline $2011-2017$ & $72.0 \%$ & $2.773(2.006-3.834)$ & $<0.001$ \\
\hline EQD2 & & & 0.183 \\
\hline$<60 \mathrm{~Gy}$ & $67.7 \%$ & & - \\
\hline$\geq 60 \mathrm{~Gy}$ & $67.9 \%$ & $0.807(0.589-1.106)$ & - \\
\hline
\end{tabular}

\section{Discussion}


Our research confirms the efficacy of concurrent chemotherapy in treating patients with locally advanced ESCC by combining with IMRT. According to the matched results, the 5-year OS of the CCRT group was as high as $38.1 \%$, and the concurrent chemotherapy increased the 1-year, 3-year, and 5-year OS rates of RT alone group by $8.9 \%, 10.6 \%$, and $9.6 \%$, and the 1 -year, 3 -year, and 5 -year PFS rates by $4.3 \%, 7.8 \%$, and $9.4 \%$, respectively. Although the incidence of toxicities also increased accordingly, the general incidence of Grade 3-4 toxicities in the CCRT group was still within the acceptable range (the most common Grade 3-4 side effects is leukopenia, the incidence of which is $13.7 \%$ ). Besides, CCRT did not increase the incidence of radiation-related pneumonitis or treatment-related mortality, indicating that for patients with locally advanced ESCC, definitive IMRT concurrent with chemotherapy are safe and tolerable.

In the subgroup analysis, for elderly patients (aged 70 years or older), CCRT did not show a significant survival advantage over RT alone. This result is consistent with some recently published retrospective analyses of elderly patients[14]. This can be related to the poor tolerance of elderly patients for CCRT. One should note that there is still lack of consensus on the optimal treatment option for elderly patients with esophageal cancer, and corresponding prospective randomized studies are needed to further explore the role of concurrent chemotherapy in elderly patients, as well as the best concurrent chemotherapy regimen and other issues.

Although since the publication of RTOG 94 - 05[15] study, 50.4 Gy became the recommended radiation dose for EC patients receiving non-surgical treatment, physicians of our center are still more accustomed to applying higher dose prescriptions. Besides, in our multivariate Cox analysis after PSM, higher radiation dose (EQD2 $\geq$ 60Gy) was found to be an independent protective prognostic factor for both OS and PFS. RTOG $94-05$ is a research conducted in the era of 2DRT. In recent years, the emergence of more advanced radiation techniques (3DCRT, IMRT, etc.) has made it possible to increase the dose of target volumes while reducing the exposure of adjacent normal tissues[10,11]. Numerous recently-published retrospective analysis studies[16, 17] and metaanalysis[18] also showed that patients with ESCC may benefit from high-dose radiotherapy. At present, a series of prospective clinical trials[19-22] applying simultaneous integrated boost-IMRT are in progress. Their results confirmed the safety and feasibility of high-dose IMRT concurrent with chemotherapy in patients with ECE, and satisfactory local control and OS has been achieved.

It is true that the proportion of patients receiving concurrent chemotherapy in this study is relatively low when compared with most contemporaneous large-scale real-world reports in developed countries[23]. Multivariate logistic regression analysis showed that the factors affecting the rate of concurrent chemotherapy were age, gender, TNM stage and year of diagnosis. In addition, we believe that this phenomenon is also related to the advanced TNM stage, large tumor burden, poor general condition, and poor economic status of patients treated in our center. However, our research also confirmed that relatively satisfactory survival rates could be achieved even with IMRT monotherapy for patients with locally advanced ESCC. In the pre-matched original sample, a total of 590 patients received IMRT monotherapy as the radical treatment, of which over $80 \%$ had stage IIIIIV disease. For patients with such advanced disease at initial diagnosis, RT alone still resulted in 1-year, 3-year and 5 -year OS rates of $65.3 \%, 32.6 \%$, and $24.8 \%$, indicating its efficacy in treating patients with advanced ESCC. Our results are consistent with other recently published researches [24,25], and show a much better survival than that with patients treated by 2DRT[7]. All these results suggest that for patients with locally advanced ESCC who cannot receive CCRT, radiation monotherapy can be an effective and safe alternative with promising survival results. 
There were several limitations associated with this study. First of all, this is a retrospective analysis. However, we mimicked randomization through propensity score matching, which eliminated potential bias by creating two comparable groups. Secondly, this was a single- institution study. However, the large sample size enhanced the reliability of the results.

\section{Conclusions}

This study confirmed the safety and efficacy of concurrent chemotherapy in patients with locally advanced ESCC receiving non-surgical treatment in the era of IMRT. For some patients who cannot receive or tolerate concurrent chemotherapy for various reasons, IMRT alone could bring promising survival results as an alternative option.

\section{Abbreviations}

PSM: propensity score matching; EC: esophageal cancer; SCC: squamous cell carcinoma; CCRT: concurrent chemoradiotherapy; 2DRT: two-dimensional radiotherapy; 3DCRT: three-dimensional conformal radiotherapy; IMRT: intensity-modulated radiotherapy; CT: computed tomography; GTV-T: gross tumor volume; GTV-N: gross lymph nodes volume; CTV: clinical target volume; PTV: planning target volume; PGTV: boost volume; EQD2: equivalent dose in $2 \mathrm{~Gy}$ /fraction; DVH: dose volume histograms; IGRT: image-guided radiotherapy; EPID:

electronic portal imaging; СBCT: cone beam computed tomography; EUS: endoscopic ultrasonography; PET-CT: positron emission computed tomography; ALP: alkaline phosphatase; OS: overall survival. PFS: progressionfree survival; ORs; odds ratios; 95\% Cl: 95\% confidence intervals; KM: Kaplan- Meier; HRs: hazard ratios; KPS: Karnofsky Performance Status

\section{Declarations}

\section{Acknowledgements}

Not applicable

\section{Authors' contributions}

Zefen Xiao designed the study and revised the article critically; Chen Li collected and analysed the data, interpreted the results and draft the article; Lijun Tan, Xiao Liu, Wenjie Ni, Xiao Chang, Weiming Han, Linrui Gao and Shijia Wang helped in the collection of data; Xin Wang, Zongmei Zhou, Dongfu Chen, Qinfu Feng, Jun Liang, Jima Lv, Xiaozhen Wang, Nan Bi, Lei Deng, Wenqing Wang, Tao Zhang were involved in study implementation. All authors gave final approval of the version to be published.

\section{Availability of data and materials}

The datasets used and/or analyzed during the current study are available from the corresponding author on reasonable request. 


\section{Authors' information}

${ }^{1}$ Department of Radiation Oncology, National Cancer Center/National Clinical Research Center for Cancer/Cancer Hospital, Chinese Academy of Medical Sciences and Peking Union Medical College, Beijing 100021, China.

${ }^{2}$ Department of Oncology, First Affiliated Hospital of Harbin Medical College, Harbin 150000, China

${ }^{3}$ Department of Radiation Oncology, Henan Cancer Hospital, Zhengzhou, 450000, China.

${ }^{4}$ Department of Radiation Oncology, Cancer Hospital Chinese Academy of Medical Sciences, Shenzhen Center, Shenzhen, 518000, China

\section{Competing interests}

The authors declare that they have no competing interests.

\section{Consent to publish}

Not applicable.

\section{Ethics approval and consent to participate}

This retrospective study was approved by Chinese Academy of Medical Sciences Institutional Review Board. All patients provided written informed consent prior to treatment, and all information was anonymized prior to analysis. All methods were carried out in accordance with relevant guidelines and regulations.

\section{Funding}

This study was supported by Beijing Hope Run Special Fund of Cancer Foundation of China (LC2016L04) and National Key Projects of Research and Development of China (2016YFC0904600).

The funding source has no role in study design, data collection, analysis, interpretation, the writing of the manuscript, or the decision to submit the current study.

\section{References}

1. Chen W, Zheng R, Baade PD, Zhang S, Zeng H, Bray F, et al. Cancer statistics in China, 2015. CA Cancer J Clin [Internet]. 2016;66:115-32. Available from: http://doi.wiley.com/10.3322/caac.21338

2. Pennathur A, Gibson MK, Jobe BA, Luketich JD. Oesophageal carcinoma. Lancet [Internet]. Elsevier Ltd; 2013;381:400-12. Available from: http://dx.doi.org/10.1016/S0140-6736(12)60643-6 
3. Arnold M, Soerjomataram I, Ferlay J, Forman D. Global incidence of oesophageal cancer by histological subtype in 2012. Gut [Internet]. 2015;64:381-7. Available from:

https://gut.bmj.com/lookup/doi/10.1136/gutjnl-2014-308124

4. Herskovic A, Martz K, Al-Sarraf M, Leichman L, Brindle J, Vaitkevicius V, et al. Combined Chemotherapy and Radiotherapy Compared with Radiotherapy Alone in Patients with Cancer of the Esophagus. N Engl J Med [Internet]. 1992;326:1593-8. Available from:

http://www.nejm.org/doi/abs/10.1056/NEJM199206113262403

5. NCCN Guidelines Panel. Esophageal and Esophagogastric Junction Cancers v. 2.2018. Nccn [Internet]. 2017; Available from: https://www.dropbox.com/s/zz62u2s3oy0p2zm/esophagealNCCN.pdf?dl=0

6. Lordick F, Mariette C, Haustermans K, Obermannová R, Arnold D. Oesophageal cancer: ESMO Clinical Practice Guidelines for diagnosis, treatment and follow-up. Ann Oncol [Internet]. 2016;27:v50-7. Available from: https://linkinghub.elsevier.com/retrieve/pii/S0923753419316473

7. De-Ren S. Ten-year follow-up of esophageal cancer treated by radical radiation therapy: analysis of 869 patients. Int J Radiat Oncol [Internet]. 1989;16:329-34. Available from:

https://linkinghub.elsevier.com/retrieve/pii/0360301689903246

8. Araújo CM, Souhami L, Gil RA, Carvalho R, Garcia JA, Froimtchuk MJ, et al. A randomized trial comparing radiation therapy versus concomitant radiation therapy and chemotherapy in carcinoma of the thoracic esophagus. Cancer [Internet]. 1991;67:2258-61. Available from:

http://www.ncbi.nlm.nih.gov/pubmed/1707338

9. Sykes A., Burt P., Slevin N., Stout R, Marrs J. Radical radiotherapy for carcinoma of the oesophagus: an effective alternative to surgery. Radiother Oncol [Internet]. 1998;48:15-21. Available from:

https://linkinghub.elsevier.com/retrieve/pii/S0167814098000371

10. Welsh J, Palmer MB, Ajani JA, Liao Z, Swisher SG, Hofstetter WL, et al. Esophageal Cancer Dose Escalation Using a Simultaneous Integrated Boost Technique. Int J Radiat Oncol [Internet]. 2012;82:468-74. Available from: http://linkinghub.elsevier.com/retrieve/pii/S0360301610034449

11. Lin SH, Wang L, Myles B, Thall PF, Hofstetter WL, Swisher SG, et al. Propensity Score-based Comparison of Long-term Outcomes With 3-Dimensional Conformal Radiotherapy vs Intensity-Modulated Radiotherapy for Esophageal Cancer. Int J Radiat Oncol [Internet]. Elsevier Inc.; 2012;84:1078-85. Available from: http://dx.doi.org/10.1016/j.ijrobp.2012.02.015

12. Services H. Common Terminology Criteria for Adverse Events v4.0 (CTCAE). Disponible en: http://evs.nci.nih.gov/ftp1/CTCAE/CTCAE_4.03_2010-06-14_QuickReference_5x7.pdf. 2010;2009.

13. D’Agostino RB. Propensity score methods for bias reduction in the comparison of a treatment to a nonrandomized control group. Stat Med [Internet]. 1998;17:2265-81. Available from: http://doi.wiley.com/10.1002/\%28SICI\%291097-0258\%2819981015\%2917\%3A19\%3C2265\%3A\%3AAIDSIM918\%3E3.0.CO\%3B2-B

14. JINGU K, TAKAHASHI N, MURAKAMI Y, ISHIKAWA K, ITASAKA S, TAKAHASHI T, et al. Is Concurrent Chemotherapy With Radiotherapy for Esophageal Cancer Beneficial in Patients Aged 80 Years or Older? Anticancer Res [Internet]. 2019;39:4279-83. Available from:

http://ar.iiarjournals.org/lookup/doi/10.21873/anticanres.13592 
15. Minsky BD, Pajak TF, Ginsberg RJ, Pisansky TM, Martenson J, Komaki R, et al. INT 0123 (Radiation Therapy Oncology Group 94-05) Phase III Trial of Combined-Modality Therapy for Esophageal Cancer: High-Dose Versus Standard-Dose Radiation Therapy. J Clin Oncol [Internet]. 2002;20:1167-74. Available from: http://ascopubs.org/doi/10.1200/JC0.2002.20.5.1167

16. Chen C-Y, Li C-C, Chien C-R. Does higher radiation dose lead to better outcome for non-operated localized esophageal squamous cell carcinoma patients who received concurrent chemoradiotherapy? A population based propensity-score matched analysis. Radiother Oncol [Internet]. Elsevier Ireland Ltd; 2016;120:136-9. Available from: http://dx.doi.org/10.1016/j.radonc.2016.04.042

17. Chang C-L, Tsai H-C, Lin W-C, Chang J-H, Hsu H-L, Chow J-M, et al. Dose escalation intensity-modulated radiotherapy-based concurrent chemoradiotherapy is effective for advanced-stage thoracic esophageal squamous cell carcinoma. Radiother Oncol [Internet]. Elsevier B.V.; 2017;125:73-9. Available from: http://dx.doi.org/10.1016/j.radonc.2017.08.025

18. Song T, Liang X, Fang M, Wu S. High-dose versus conventional-dose irradiation in cisplatin-based definitive concurrent chemoradiotherapy for esophageal cancer: a systematic review and pooled analysis. Expert Rev Anticancer Ther [Internet]. Informa UK, Ltd.; 2015;15:1157-69. Available from: http://dx.doi.org/10.1586/14737140.2015.1074041

19. Welsh JW, Seyedin SN, Allen PK, Hofstetter WL, Ajani JA, Chang JY, et al. Local Control and Toxicity of a Simultaneous Integrated Boost for Dose Escalation in Locally Advanced Esophageal Cancer: Interim Results from a Prospective Phase I/II Trial. J Thorac Oncol [Internet]. Elsevier Ltd; 2017;12:375-82. Available from: http://dx.doi.org/10.1016/j.jtho.2016.10.013

20. Chen D, Menon H, Verma V, Seyedin SN, Ajani JA, Hofstetter WL, et al. Results of a Phase 1/2 Trial of Chemoradiotherapy With Simultaneous Integrated Boost of Radiotherapy Dose in Unresectable Locally Advanced Esophageal Cancer. JAMA Oncol [Internet]. 2019;5:1597. Available from:

https://jamanetwork.com/journals/jamaoncology/fullarticle/2751404

21. Yu W, Cai X-W, Liu Q, Zhu Z-F, Feng W, Zhang Q, et al. Safety of dose escalation by simultaneous integrated boosting radiation dose within the primary tumor guided by 18FDG-PET/CT for esophageal cancer. Radiother Oncol [Internet]. Elsevier Ireland Ltd; 2015;114:195-200. Available from: http://dx.doi.org/10.1016/j.radonc.2014.12.007

22. Yu W-W, Zhu Z-F, Fu X-L, Zhao K-L, Mao J-F, Wu K-L, et al. Simultaneous integrated boost intensitymodulated radiotherapy in esophageal carcinoma. Strahlentherapie und Onkol [Internet]. 2014;190:97986. Available from: http://link.springer.com/10.1007/s00066-014-0636-y

23. Tachimori Y, Ozawa S, Numasaki H, Ishihara R, Matsubara H, Muro K, et al. Comprehensive registry of esophageal cancer in Japan, 2012. Esophagus [Internet]. Springer Singapore; 2019;16:221-45. Available from: https://doi.org/10.1007/s10388-019-00674-z

24. Wu K-L, Chen G-Y, Xu Z-Y, Fu X-L, Qian H, Jiang G-L. Three-dimensional conformal radiation therapy for squamous cell carcinoma of the esophagus: A prospective phase I/II study. Radiother Oncol [Internet]. Elsevier Ireland Ltd; 2009;93:454-7. Available from: http://dx.doi.org/10.1016/j.radonc.2009.10.014

25. Fan XW, Wu JL, Wang HB, Liang F, Jiang GL, Wu KL. Three-dimensional conformal radiation therapy alone for esophageal squamous cell carcinoma: 10-year survival outcomes. Thorac Cancer. 2019;10:519-25. 


\section{Figures}

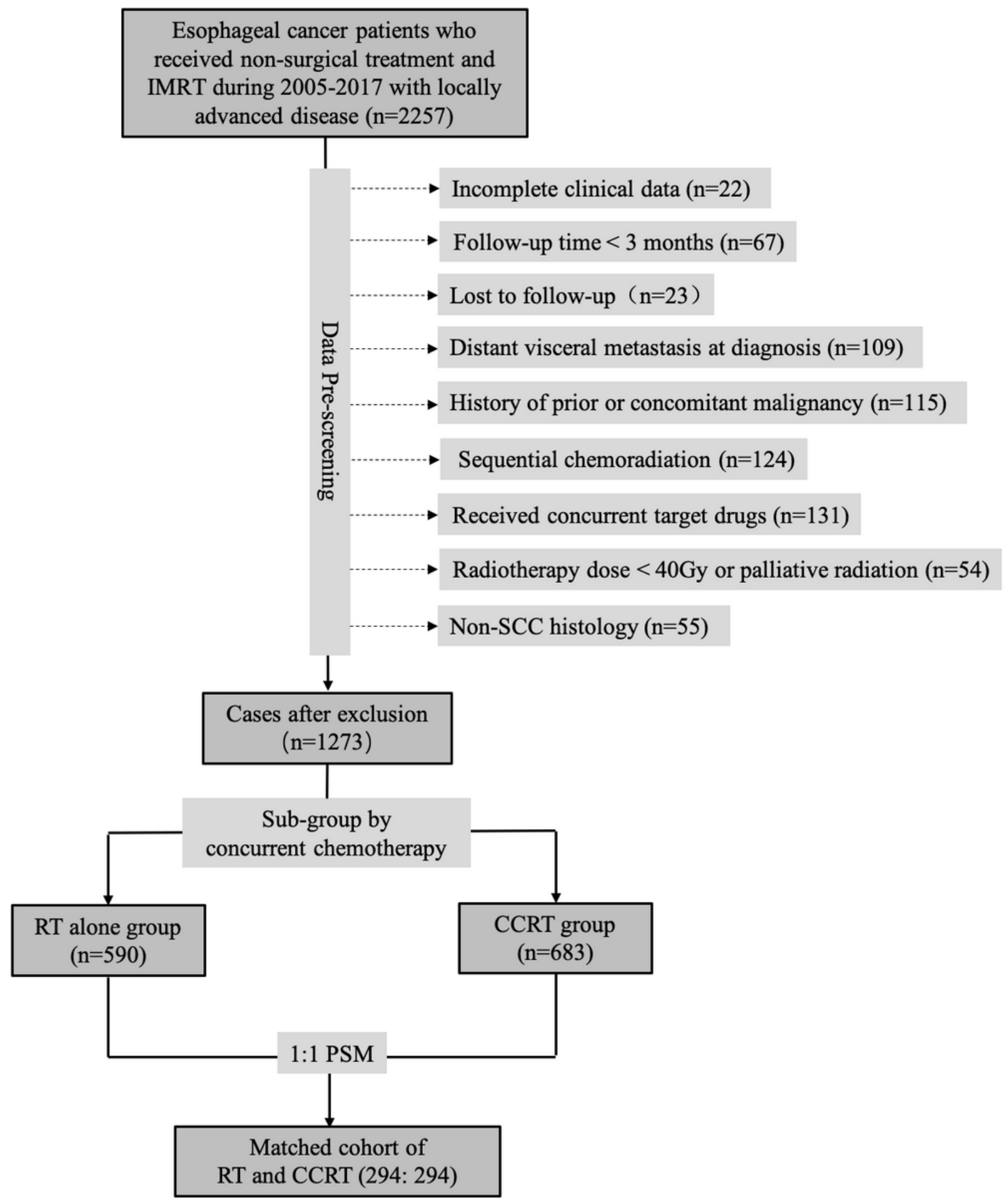

Figure 1

CONSORT diagram showing patient selection. RT: radiotherapy; CCRT: concurrent chemoradiotherapy; PSM, propensity score matching. 


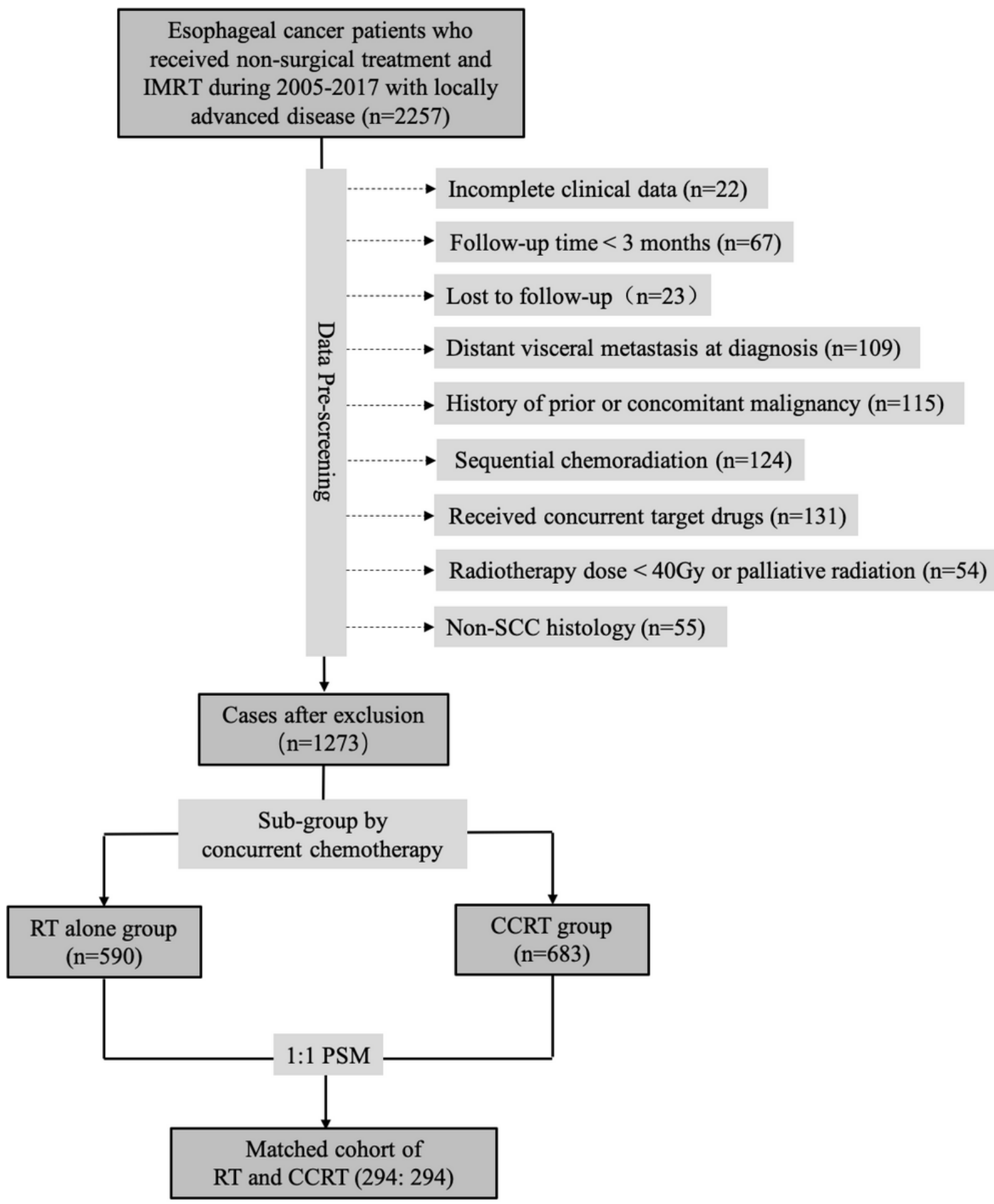

Figure 1

CONSORT diagram showing patient selection. RT: radiotherapy; CCRT: concurrent chemoradiotherapy; PSM, propensity score matching. 

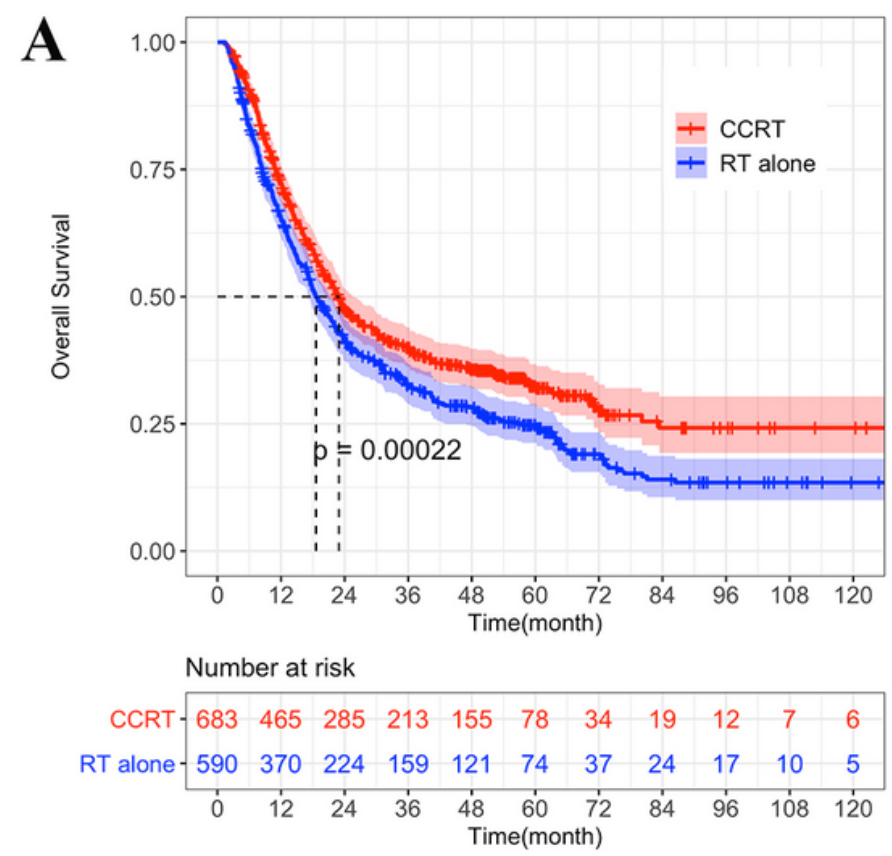

C
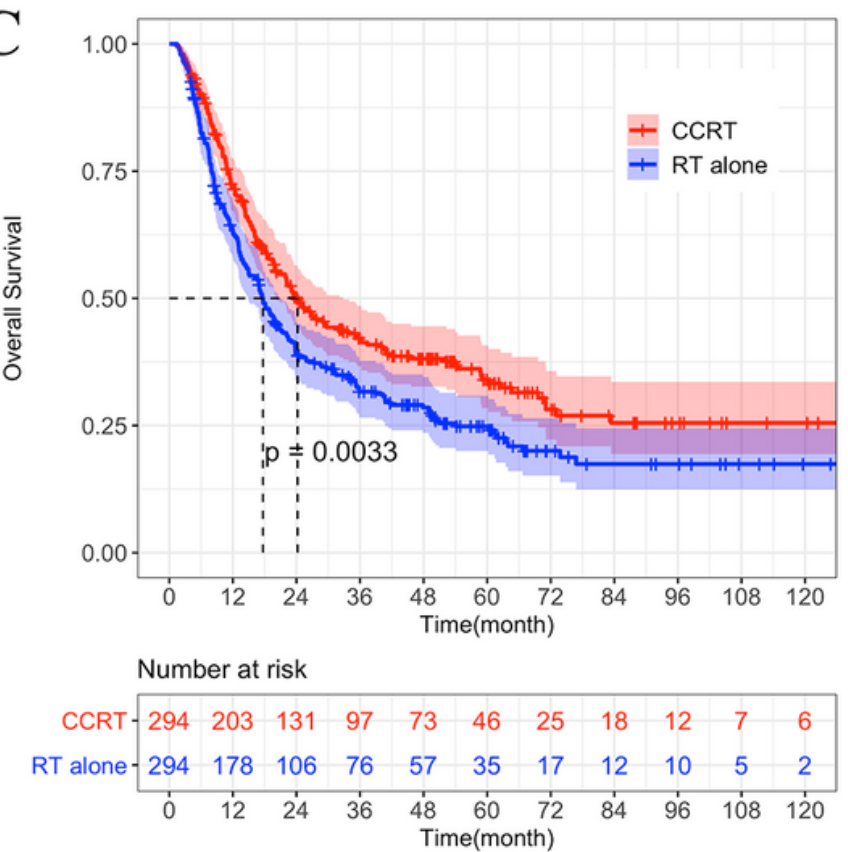
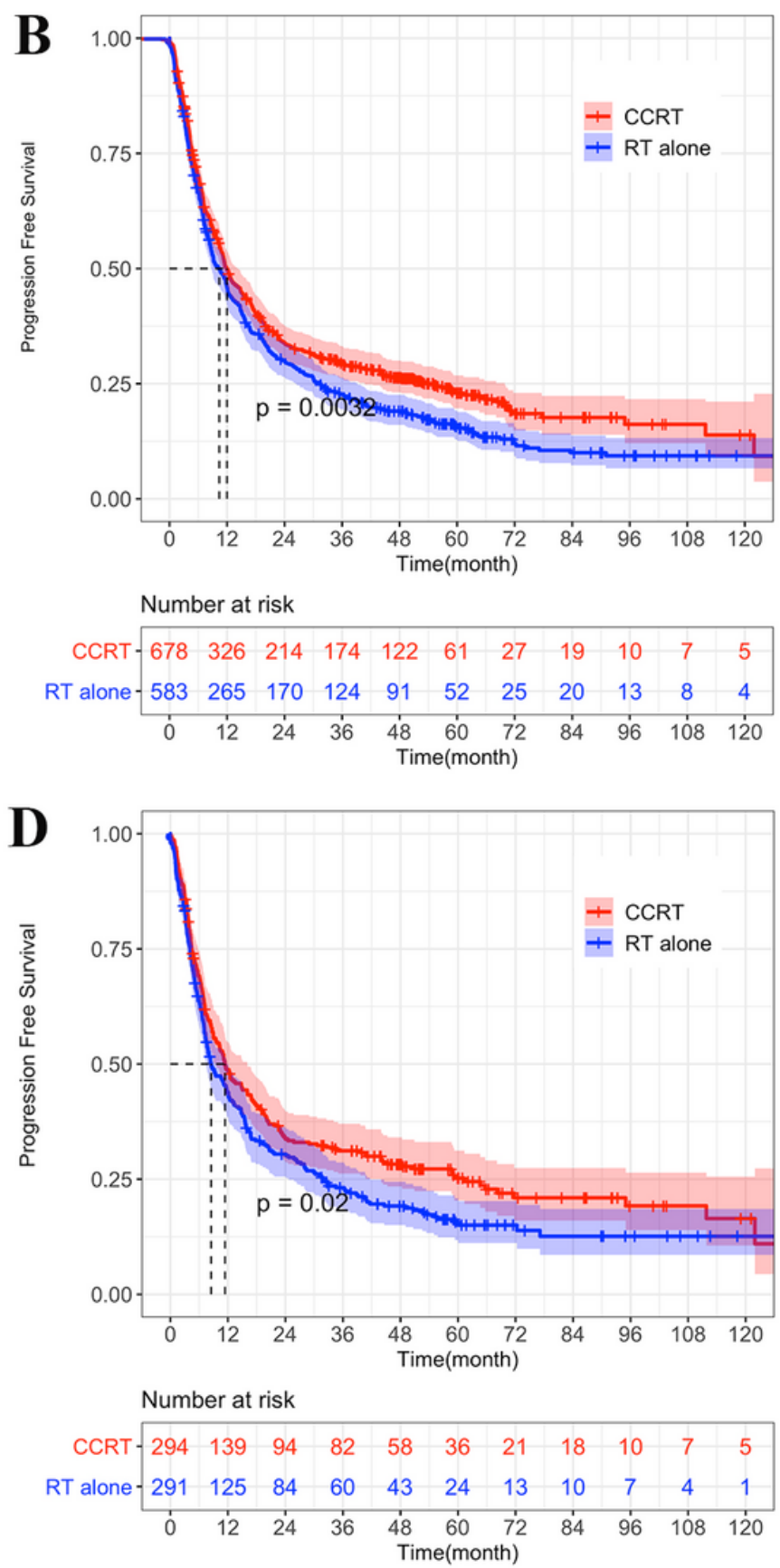

\section{Figure 2}

OS and PFS of patients receiving RT and CCRT both before and after PSM. Kaplan-Meier (KM) estimates of (A) OS before PSM, with 95\% Cls. (B) OS after PSM, with 95\% Cls. (C) PFS before PSM, with 95\% Cls. (D) PFS after PSM, with 95\% Cls. Blue curve represents survival in the RT alone group; red curve, survival in the CCRT group. 

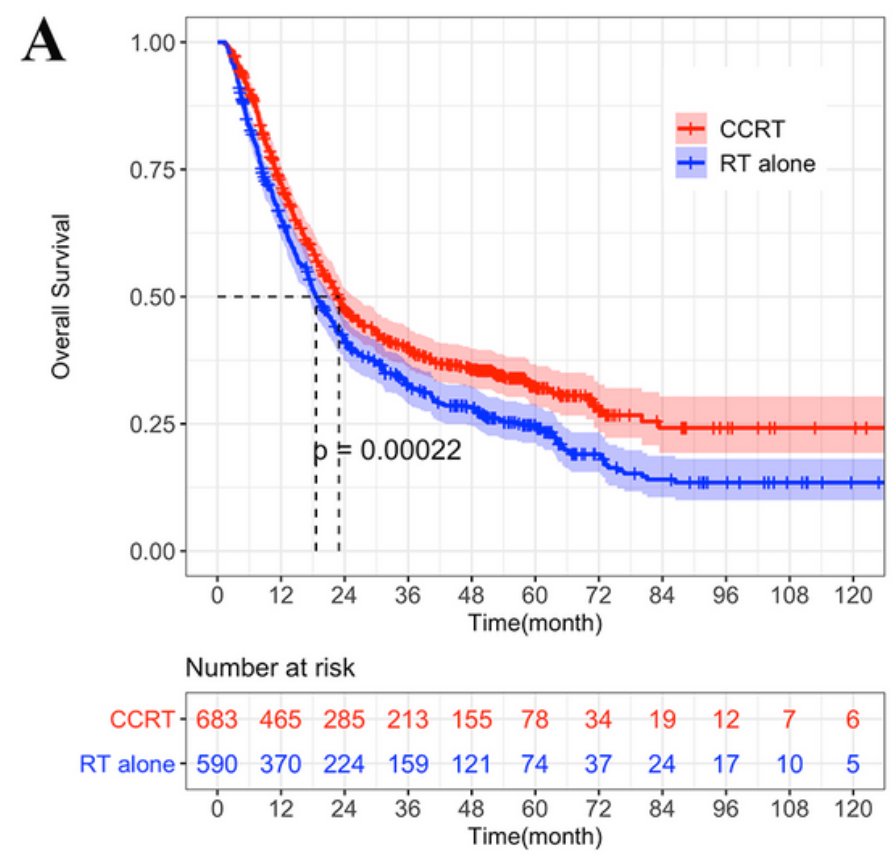

C
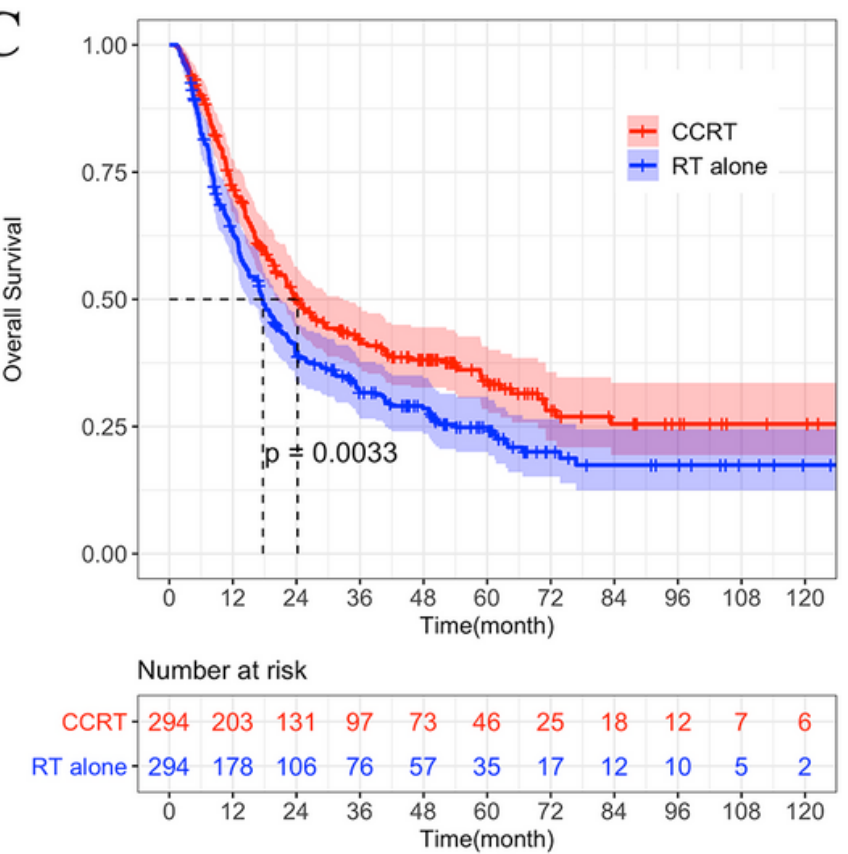
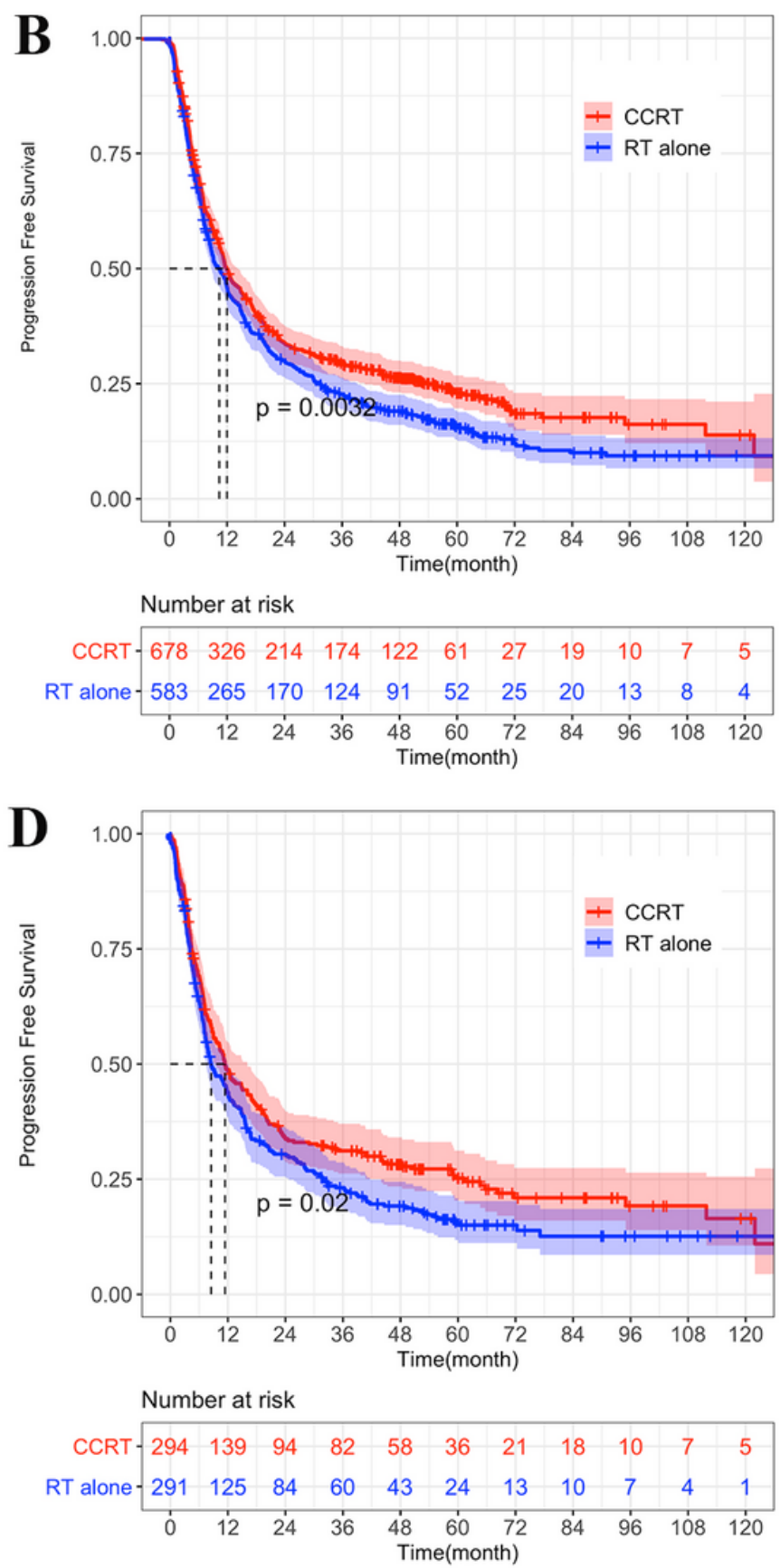

\section{Figure 2}

OS and PFS of patients receiving RT and CCRT both before and after PSM. Kaplan-Meier (KM) estimates of (A) OS before PSM, with 95\% Cls. (B) OS after PSM, with 95\% Cls. (C) PFS before PSM, with 95\% Cls. (D) PFS after PSM, with 95\% Cls. Blue curve represents survival in the RT alone group; red curve, survival in the CCRT group. 

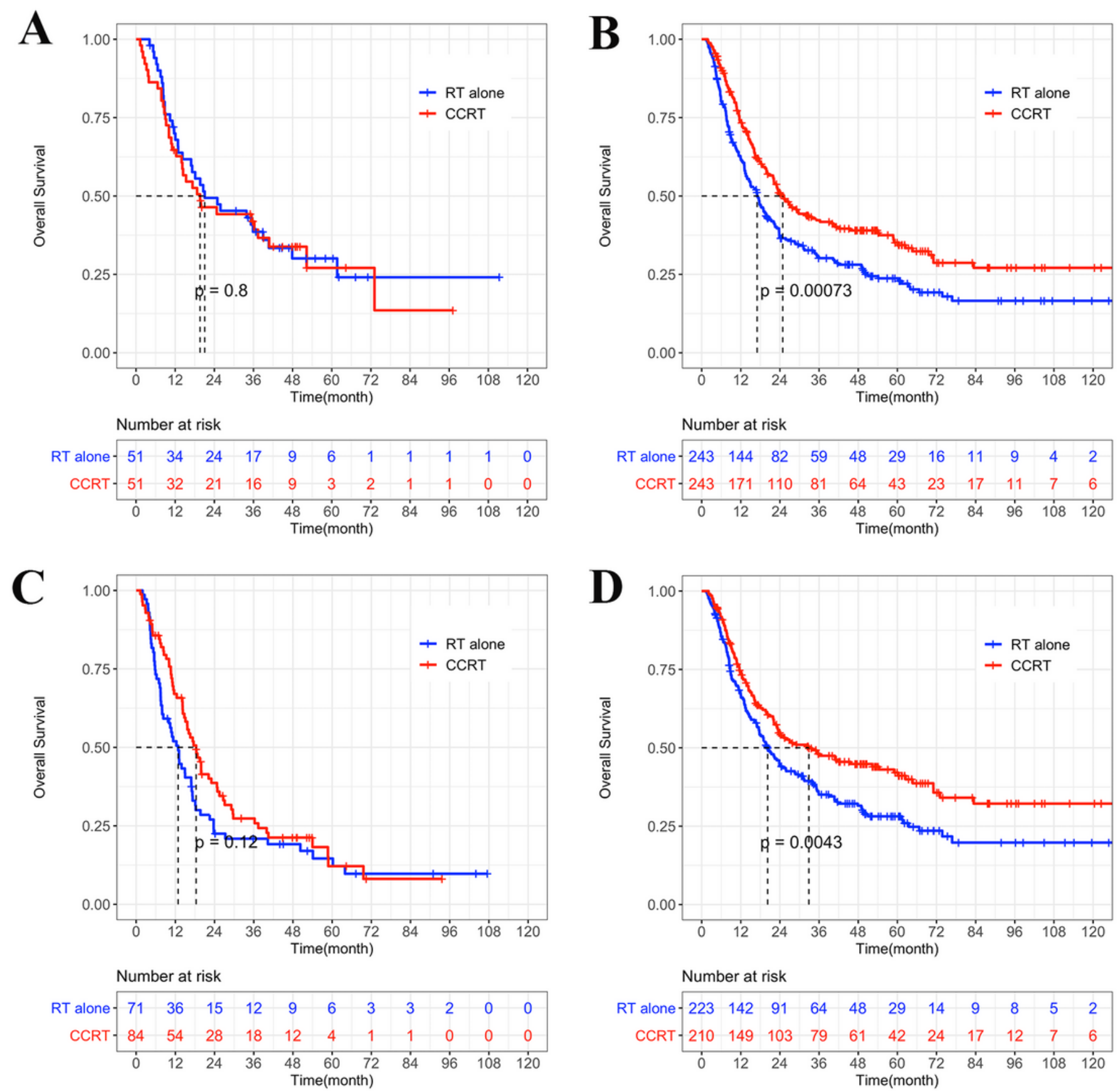

\section{Figure 3}

Subgroup analysis of patients receiving RT and CCRT in the after-PSM sample. Kaplan-Meier (KM) estimates of OS of (A) Elderly patients ( $\geq 70$ years old). (B) Non-elderly patients ( $<70$ years old). (C) Low-dose radiation group (EQD2 <60Gy) and (D) High-dose radiation group (EQD2 $\geq 60 \mathrm{~Gy}$ ). Blue curve represents survival in the RT alone group; red curve, survival in the CCRT group. 

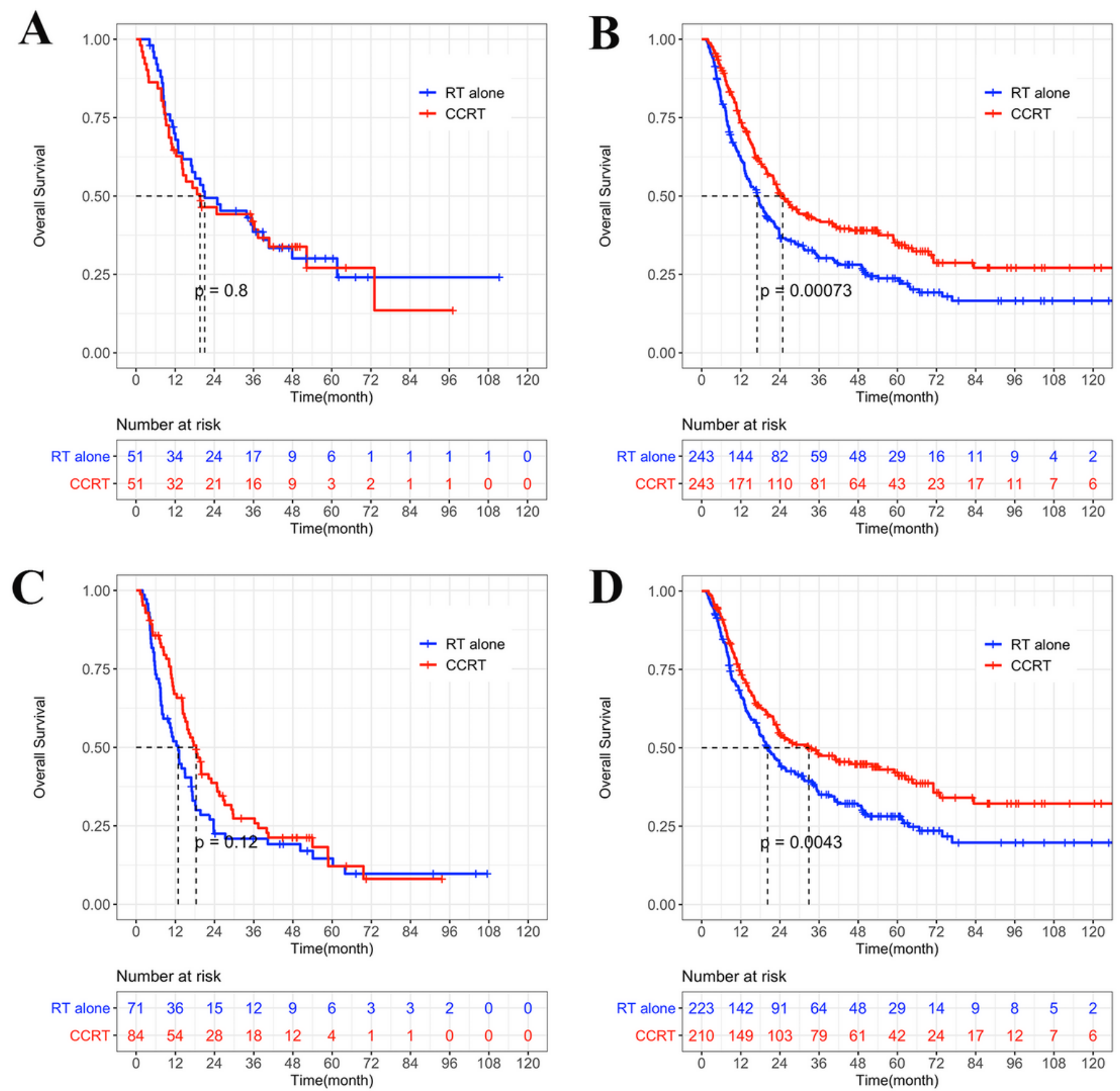

\section{Figure 3}

Subgroup analysis of patients receiving RT and CCRT in the after-PSM sample. Kaplan-Meier (KM) estimates of OS of (A) Elderly patients ( $\geq 70$ years old). (B) Non-elderly patients ( $<70$ years old). (C) Low-dose radiation group (EQD2 <60Gy) and (D) High-dose radiation group (EQD2 $\geq 60 \mathrm{~Gy}$ ). Blue curve represents survival in the RT alone group; red curve, survival in the CCRT group. 